\title{
GRB host galaxies: theoretical investigation
}

\author{
Jirong Mao \\ Korea Astronomy and Space Science Institute 776, Daedeokdae-ro, Yuseong-gu, Daejeon, \\ Republic of Korea 305-348 \\ email: jirongmao@kasi.re.kr
}

\begin{abstract}
Long gamma-ray bursts (GRBs) can be linked to the massive stars and their host galaxies are assumed to be the star-forming galaxies within small dark matter halos. We apply a galaxy evolution model, in which the star formation process inside the virialized dark matter halo at a given redshift is achieved. The star formation rates (SFRs) in the GRB host galaxies at different redshifts can be derived from our model. The related stellar masses, luminosities, and metalicities of these GRB host galaxies are estimated. We also calculate the X-ray and optical absorption of GRB afterglow emission. At higher redshift, the SFR of host galaxy is stronger, and the absorption in the X-ray and optical bands of GRB afterglow is stronger, when the dust and metal components are locally released, surrounding the GRB environment. These model predictions are compared with some observational data as well.
\end{abstract}

Keywords. gamma rays: bursts, galaxies: high-redshift, dust, extinction.

\section{Introduction}

Long-duration gamma-ray burst (GRB) progenitors have been proposed as massive collapsing stars. Some oflong GRBs can be found in star formation galaxies which are dominated by young stellar populations. GRBs favor a metal-poor environment. Moreover, those high-redshift $(z>2)$ GRB host galaxies may trace the star formation of the universe. The high global star formation rate (SFR) history at redshifts larger than 6 indicate the possibility of high-redshift GRB production and the detection of host galaxies. On the other hand, the heavy attenuation shown in X-ray afterglow has been given, indicating a dense surrounding environment for those GRBs. It is also interesting to understand whether this kind of strong attenuation intrinsically evolves with redshift. The characteristics of the corresponding absorption shown in optical afterglow are still under debate. In order to have an explanation of GRB afterglow obscuration, the physical origin associated with star formation and galactic evolution should be studied in an unified scenario.

\section{Results}

We specify one physical model of star-forming and metal-poor galaxies as the hosts of long GRBs, exploiting the physical recipes from Granato et al. (2004). In the general scenario at each redshift bin, the SFR and galaxy mass in the given dark halo potential well have been calculated. In particular, Mao et al. (2007) have calculated the UV luminosities and the relative dust attenuation in the star-forming and metal-poor galaxies. The results are given in Fig. 1-Fig. 6. We invite readers to see the paper of mao (2010) for the physical processes and the denotations shown in the plots in detail. We note that our theoretical results from the model with dark matter halo mass of $5 \times 10^{11} M_{\odot}$ and the corresponding galactic evolutionary timescale of $5.0 \times 10^{7}$ yr are consistent with those observational data well. 


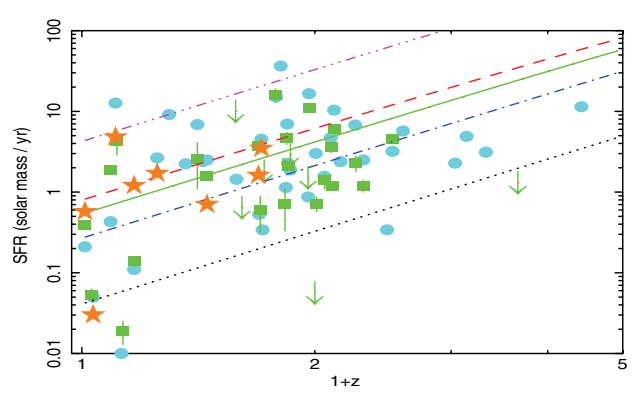

Figure 1. SFR of host

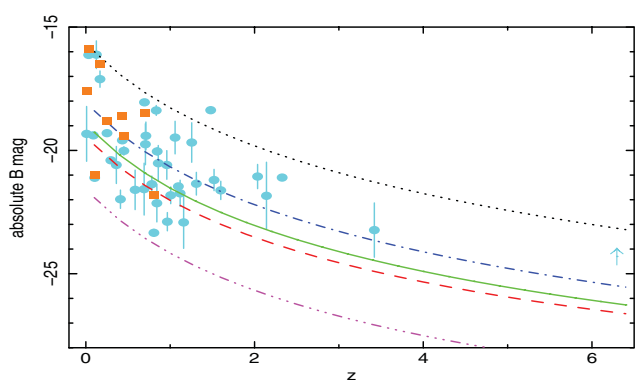

Figure 3. B-band luminosity of host

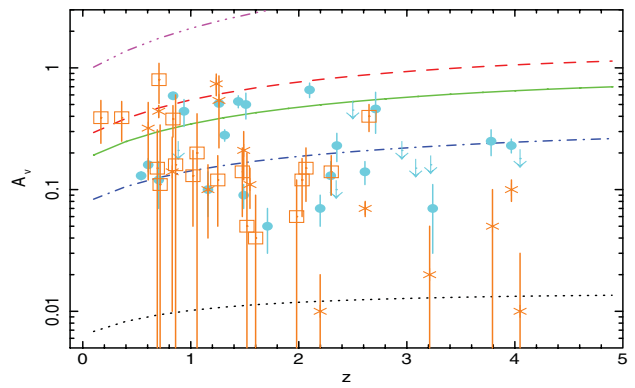

Figure 5. dust absorption of optical afterglow

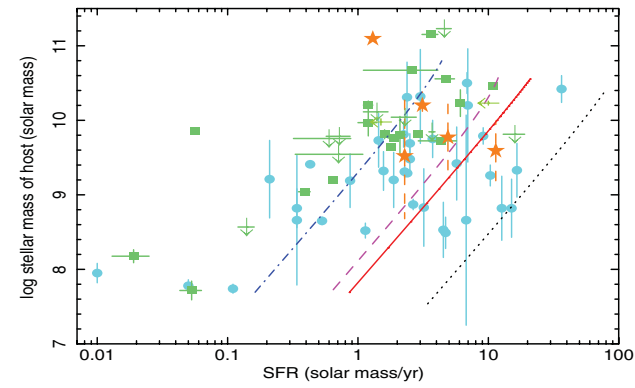

Figure 2. Stellar mass vs. SFR

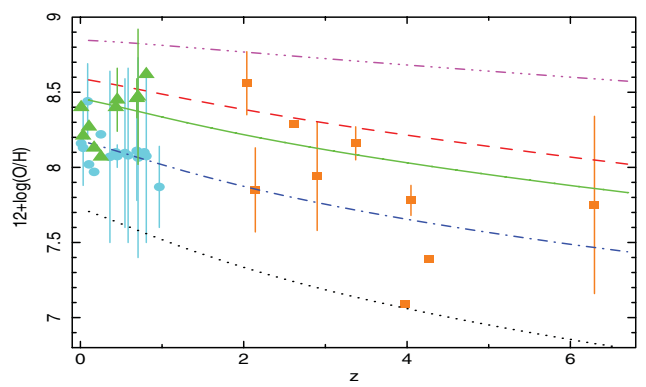

Figure 4. metallicity of host

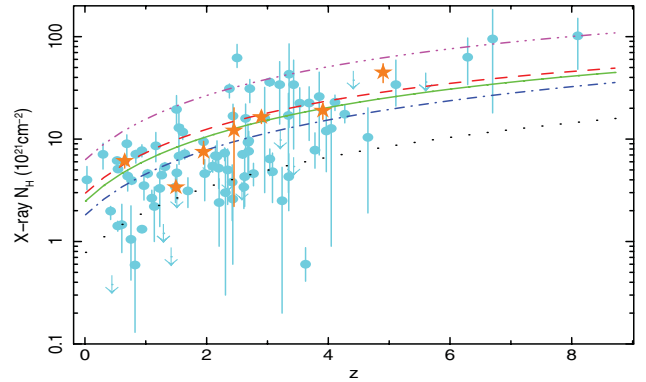

Figure 6. attenuation of X-ray afterglow

\section{Conclusion}

We reveal that some properties from afterglow emissions and GRB hosts have shown a possible intrinsic cosmological evolution even some selection effects are considered. We speculate that improving the sensitivity of detectors on high-energy telescopes is not highly useful for catching more high-redshift but faint GRBs, since low-energy-released GRBs are almost absent in the high-redshift universe. We also caution that those highredshift $(z>5)$ GRB host galaxies have not been significantly detected by space and ground-based telescopes (see Basa et al. 2012 and Tanvir et al. 2012 in detail).

\section{References}

Basa, S., et al. 2012, arXiv: 1201.6383

Granato, G. L., De Zotti, G., Silva, L., Bressan, A., \& Danese, L. 2004, ApJ, 600, 580

Mao, J., Lapi, A., Granato, G. L., de Zotti, G., \& Danese, L. 2007, ApJ, 667, 655

Mao, J. 2010, ApJ, 717, 140

Tanvir, N. R., et al. 2012, arXiv: 1201.6074 